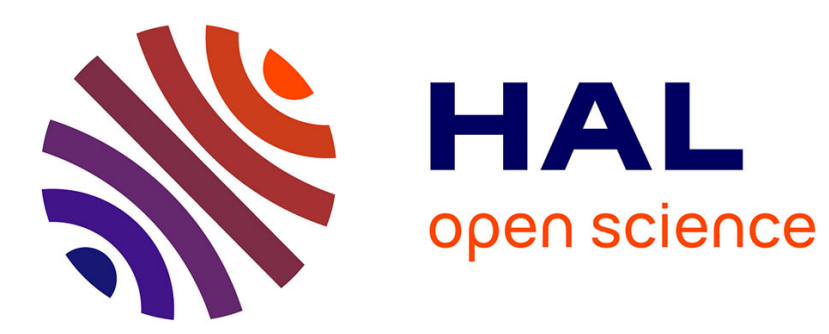

\title{
Delay-Adaptive Predictor Feedback for Systems With Unknown Long Actuator Delay
}

Delphine Bresch-Pietri, Miroslav Krstic

\section{To cite this version:}

Delphine Bresch-Pietri, Miroslav Krstic. Delay-Adaptive Predictor Feedback for Systems With Unknown Long Actuator Delay. IEEE Transactions on Automatic Control, 2010, 55 (9), pp.2106-2112. hal-00951846

\section{HAL Id: hal-00951846 \\ https://hal.science/hal-00951846}

Submitted on 26 Feb 2014

HAL is a multi-disciplinary open access archive for the deposit and dissemination of scientific research documents, whether they are published or not. The documents may come from teaching and research institutions in France or abroad, or from public or private research centers.
L'archive ouverte pluridisciplinaire HAL, est destinée au dépôt et à la diffusion de documents scientifiques de niveau recherche, publiés ou non, émanant des établissements d'enseignement et de recherche français ou étrangers, des laboratoires publics ou privés. 


\title{
Delay-Adaptive Predictor Feedback for Systems With Unknown Long Actuator Delay
}

\author{
Delphine Bresch-Pietri and Miroslav Krstic
}

\begin{abstract}
Stabilization of an unstable system with an unknown actuator delay of substantial length is an important problem that has never been attempted. We present a Lyapunovbased adaptive control design, prove its stability and regulation properties for the plant and actuator states, and present a simulation example inspired by the problem of control of pitch and flight path rates in the unstable $X-29$ aircraft.
\end{abstract}

\section{INTRODUCTION}

Adaptive control in the presence of actuator delays is challenging. Examples of existing results include [3], [9], [10]. All the existing results deal with unknown parameters but known delay. In this paper we address the more challenging problem where the delay itself is unknown and arbitrarily long.

Consider the system

$$
\dot{X}(t)=A X(t)+B U(t-D),
$$

where $X \in \mathbb{R}^{n}$ and where the delay length $D$ is unknown (though constant) and arbitrarily large. We use an actuator delay model given by a transport PDE,

$$
\begin{aligned}
\dot{X}(t) & =A X(t)+B u(0, t) \\
D u_{t}(x, t) & =u_{x}(x, t) \\
u(1, t) & =U(t),
\end{aligned}
$$

where $u(x, t)$ is the state of the actuator. Instead of a full-state measurement of the actuator state, $u(x, t)=U(t+D(x-1))$, we employ the state estimate

$$
\hat{u}(x, t)=U(t+\hat{D}(t)(x-1)),
$$

where $\hat{D}(t)$ is the estimate of the unknown delay, obtaining the following transport equation representation:

$$
\begin{aligned}
\hat{D}(t) \hat{u}_{t}(x, t) & =\hat{u}_{x}(x, t)+\dot{\hat{D}}(t)(x-1) \hat{u}_{x}(x, t) \\
\hat{u}(1, t) & =U(t) .
\end{aligned}
$$

Note that we don't use the infinite-dimensional observer (6) but only the static estimate (5), where only $\hat{D}(t)$ is updated. Let $\tilde{u}(x, t) \triangleq u(x, t)-\hat{u}(x, t)$, which yields $\dot{X}(t)=A X(t)+$ $B \hat{u}(0, t)+B \tilde{u}(0, t)$.

We employ an adaptive predictor feedback

$$
U(t)=K\left(\mathrm{e}^{A \hat{D}(t)} X(t)+\hat{D}(t) \int_{0}^{1} \mathrm{e}^{A \hat{D}(t)(1-y)} B \hat{u}(y, t) d y\right),
$$

where $K$ is selected to make the matrix $A+B K$ Hurwitz, which means, in particular, that, for any $Q=Q^{T}>0$ there exists $P=P^{T}>0$ such that $P(A+B K)+(A+B K)^{T} P=-Q$.

This work was supported by NSF and Bosch.

The authors are with the Department of Mechanical and Aerospace Engineering, University of California, San Diego, La Jolla, CA 92093-0411, USA, krsticaucsd.edu
A crucial element of our design and analysis is the backstepping transformation

$$
\begin{aligned}
\hat{w}(x, t)= & \hat{u}(x, t)-\hat{D}(t) \int_{0}^{x} K \mathrm{e}^{A \hat{D}(t)(x-y)} B \hat{u}(y, t) d y \\
& -K \mathrm{e}^{A \hat{D}(t) x} X(t) \\
\hat{u}(x, t)= & \hat{w}(x, t)+\hat{D}(t) \int_{0}^{x} K \mathrm{e}^{(A+B K) \hat{D}(t)(x-y)} B \hat{w}(y, t) d y \\
& +K \mathrm{e}^{(A+B K) \hat{D}(t) x} X(t) .
\end{aligned}
$$

Contribution and Organization. In a companion conference paper [6] we established a global adaptive result when $u(x, t)$ is measured. In this paper we establish a local result when $u(x, t)$ is replaced by the estimate $\hat{u}(x, t)$. The local adaptive result, established in Section III, builds upon robustness of predictor feedback with respect to small errors in $D$, which is shown in Section II. Simulations for an unstable scalar plant inspired by the X-29 aircraft are shown in Section IV.

\section{Nonadaptive Robustness to Delay ERror}

We take $\hat{D}(t)$ as constant establish the robustness of the predictor feedback to a small error $|D-\hat{D}|$ in an appropriate norm in which the adaptive problem will also be studied in Section III (this is a higher norm than the one in which we established delay-robustness in [4]). We denote $\omega(\theta)=$ $U(\theta), \theta \in[-\max \{D, \hat{D}\}, 0]$ and use $|\cdot|$ for a vector 2-norm.

Theorem 1: Consider the system (2)-(4), (6)-(7), (8). There exists $\delta^{*}>0$ such that for any $|\tilde{D}|<\delta^{*}$, i.e., for any $\hat{D} \in$ $\left(D-\delta^{*}, D+\delta^{*}\right)$, the zero solution of the system $(X, u, \hat{u})$ is exponentially stable, namely, there exist $R, \rho>0$ such that for all initial conditions satisfying $\left(X_{0}, u_{0}, \hat{u}_{0}\right) \in \mathbb{R}^{n} \times L_{2}(0,1) \times$ $H_{1}(0,1)$, the following holds:

$$
\begin{gathered}
\Gamma(t) \leq R \Gamma(0) \mathrm{e}^{-\rho t} \\
\Gamma(t)=|X(t)|^{2}+\int_{0}^{1}\left[u(x, t)^{2}+\hat{u}(x, t)^{2}+\hat{u}_{x}(x, t)^{2}\right] d x . \\
\text { Corollary 2: Consider system (1) with the controller } \\
U(t)=K\left(\mathrm{e}^{A \hat{D}} X(t)+\int_{t-\hat{D}}^{t} \mathrm{e}^{A(t-\theta)} B U(\theta) d \theta\right) .
\end{gathered}
$$

There exists $\delta^{*}>0$ such that, for any $|\tilde{D}|=|D-\hat{D}|<$ $\delta^{*}$, there exists $R^{\prime}>0$ such that for all $X_{0} \in R^{n}, \omega \in$ $L_{2}[-\max \{D, \hat{D}\}, 0] \cap H_{1}[-\hat{D}, 0]$ the following holds

$$
\Pi(t) \leq R^{\prime} \Pi(0) \mathrm{e}^{-\rho t}
$$

$$
\Pi(t)=|X(t)|^{2}+\int_{t-\max \{D, \hat{D}\}}^{t} U(\theta)^{2} d \theta+\int_{t-\hat{D}}^{t} \dot{U}(\theta)^{2} d \theta .
$$

We prove the theorem using the following lemmas.

Lemma 3: The system (2)-(4), (6)-(7), (8) is equivalent to the system in which the $X$-subsystem is represented as

$$
\dot{X}(t)=(A+B K) X(t)+B \hat{w}(0, t)+B \tilde{u}(0, t),
$$




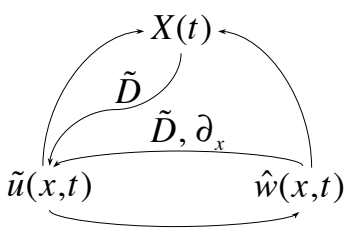

Fig. 1. Interconnections between the different variables

the $\tilde{u}$-subsystem is represented as

$$
\begin{aligned}
D \tilde{u}_{t}(x, t) & =\tilde{u}_{x}(x, t)-\tilde{D} r(x, t) \\
\tilde{u}(1, t) & =0
\end{aligned}
$$

with

$$
\begin{aligned}
r(x, t)= & K e^{(A+B K) \hat{D} x}(A+B K) X(t)+\frac{\hat{w}_{x}(x, t)}{\hat{D}}+K B \hat{w}(x, t) \\
& +\hat{D} \int_{0}^{x} K(A+B K) \mathrm{e}^{(A+B K) \hat{D}(x-y)} B \hat{w}(y, t) d y,
\end{aligned}
$$

and the $\hat{w} / \hat{w}_{x}$-subsystem is represented as

$$
\begin{aligned}
\hat{D} \hat{w}_{t}(x, t) & =\hat{w}_{x}(x, t)-\hat{D} K e^{A \hat{D} x} B \tilde{u}(0, t) \\
\hat{w}(1, t) & =0 \\
\hat{D} \hat{w}_{x t}(x, t) & =\hat{w}_{x x}(x, t)-\hat{D}^{2} K \mathrm{e}^{A \hat{D} x} A B \tilde{u}(0, t) \\
\hat{w}_{x}(1, t) & =\hat{D} K \mathrm{e}^{A \hat{D}} \tilde{u}(0, t) .
\end{aligned}
$$

The $(X, \tilde{u}, \hat{w})$-system is shown in Figure 1 . The $\tilde{D}$ connections are 'weak' and disappear when $\hat{D}=D$. The exponentially stable cascade connections $\tilde{u} \rightarrow X$ and $\tilde{u} \rightarrow$ $\hat{w} \rightarrow X$ are 'strong' and present even when $\hat{D}=D$. The potentially destabilizing feedback connections through $\tilde{D}$ can be suppressed by making $\tilde{D}$ small. An additional difficulty is that an 'unbounded' connection from $\hat{w}_{x}$ to $\tilde{u}$ exists. We deal with it by including an $H_{1}$ norm in the stability analysis.

Lemma 4: The following holds for (9) and (10):

$$
\begin{aligned}
\hat{u}_{x}(x, t)= & \hat{w}_{x}(x, t)+\hat{D} K B \hat{w}(x, t) \\
& +\hat{D} \int_{0}^{x} K(A+B K) \hat{D} \mathrm{e}^{(A+B K) \hat{D}(x-y)} B \hat{w}(y, t) d y \\
& +K(A+B K) \hat{D} \mathrm{e}^{(A+B K) \hat{D} x} X(t) \\
\hat{w}_{x}(x, t)= & \hat{u}_{x}(x, t)+\hat{D} K B \hat{u}(x, t)+\hat{D} \int_{0}^{x} K A \hat{D} \mathrm{e}^{A \hat{D}(x-y)} B \hat{u}(y, t) d y \\
& +K A \hat{D} \mathrm{e}^{A \hat{D} x} X(t) .
\end{aligned}
$$

Lemma 5: The following holds for (9), (10), (24), (25):

$$
\begin{aligned}
\|\hat{u}(t)\|^{2} & \leq p_{1}\|\hat{w}(t)\|^{2}+p_{2}|X(t)|^{2} \\
\left\|\hat{u}_{x}(t)\right\|^{2} & \leq 4\left\|\hat{w}_{x}(t)\right\|^{2}+p_{3}\|\hat{w}(t)\|^{2}+p_{4}|X(t)|^{2} \\
\|\hat{w}(t)\|^{2} & \leq q_{1}\|\hat{u}(t)\|^{2}+q_{2}|X(t)|^{2} \\
\left\|\hat{w}_{x}(t)\right\|^{2} & \leq 4\left\|\hat{u}_{x}(t)\right\|^{2}+q_{3}\|\hat{u}(t)\|^{2}+q_{4}|X(t)|^{2},
\end{aligned}
$$

where

$$
\begin{aligned}
& p_{1}(\hat{D})=3\left(1+\hat{D}^{2}|K|^{2} \mathrm{e}^{2|A+B K| \hat{D}}|B|^{2}\right) \\
& p_{2}(\hat{D})=3|K|^{2} \mathrm{e}^{2|A+B K| \hat{D}} \\
& p_{3}(\hat{D})=4 \hat{D}^{2}|K|^{2}|B|^{2}\left(1+\hat{D}^{2}|A+B K|^{2} \mathrm{e}^{2 \hat{D}|A+B K|}\right) \\
& p_{4}(\hat{D})=4|K|^{2} \hat{D}^{2}|A+B K|^{2} \mathrm{e}^{2 \hat{D}|A+B K|} \\
& q_{1}(\hat{D})=3\left(1+\hat{D}^{2}|K|^{2} \mathrm{e}^{2|A| \hat{D}}|B|^{2}\right)
\end{aligned}
$$

$$
\begin{aligned}
& q_{2}(\hat{D})=3|K|^{2} \mathrm{e}^{2|A| \hat{D}} \\
& q_{3}(\hat{D})=4 \hat{D}^{2}|K|^{2}|B|^{2}\left(1+\hat{D}^{2}|A|^{2} \mathrm{e}^{2 \hat{D}|A|}\right) \\
& q_{4}(\hat{D})=4|K|^{2} \hat{D}^{2}|A|^{2} \mathrm{e}^{2 \hat{D}|A|}
\end{aligned}
$$

Lemma 6: Consider the Lyapunov function

$$
\begin{aligned}
& V(t)=X^{T}(t) P X(t)+b_{1} D \int_{0}^{1}(1+x) \tilde{u}(x, t)^{2} d x \\
& +b_{2} \hat{D}\left(\int_{0}^{1}(1+x) \hat{w}(x, t)^{2} d x+\int_{0}^{1}(1+x) \hat{w}_{x}(x, t)^{2} d x\right) .
\end{aligned}
$$

There exist positive constants $b_{1}, b_{2}, \rho$, and $\delta^{*}$ such that for any $|\tilde{D}|<\delta^{*}$ the following holds

$$
\dot{V} \leq-\rho V \text {. }
$$

Proof: Differentiating (38), along the solutions of (16), (17), (20), (22), and using integration by parts, we obtain

$$
\begin{aligned}
\dot{V}= & -X^{T}(t) Q X(t)+2 X^{T}(t) P B(\hat{w}(0, t)+\tilde{u}(0, t)) \\
& +2 b_{1} D \int_{0}^{1}(1+x) \tilde{u}(x, t) \tilde{u}_{t}(x, t) d x \\
& +2 b_{2} \hat{D}\left(\int_{0}^{1}(1+x) \hat{w}(x, t) \hat{w}_{t}(x, t) d x\right. \\
& \left.+\int_{0}^{1}(1+x) \hat{w}_{x}(x, t) \hat{w}_{x t}(x, t) d x\right) \\
= & -X^{T} Q X+2 X^{T} P B(\hat{w}(0, t)+\tilde{u}(0, t)) \\
& -2 b_{1}\left(\frac{\tilde{u}(0, t)^{2}}{2}+\frac{\|\tilde{u}(t)\|^{2}}{2}+\tilde{D} \int_{0}^{1}(1+x) \tilde{u}(x, t) r(x, t) d x\right) \\
& -2 b_{2}\left(\frac{\hat{w}(0, t)^{2}}{2}+\frac{\|\hat{w}(t)\|^{2}}{2}\right. \\
& \left.+\hat{D}_{K} \int_{0}^{1}(1+x) \hat{w}(x, t) \mathrm{e}^{A \hat{D} x} \tilde{u}(0, t) d x\right) \\
& -2 b_{2}\left(\frac{\hat{w}_{x}(0, t)^{2}}{2}-\hat{w}_{x}(1, t)^{2}+\frac{\left\|\hat{w}_{x}(t)\right\|^{2}}{2}\right. \\
& \left.+\hat{D}^{2} K A \int_{0}^{1}(1+x) \hat{w}_{x}(x, t) \mathrm{e}^{A \hat{D} x} B \tilde{u}(0, t) d x\right) .
\end{aligned}
$$

Let us define the following constants, $c_{1}=K\|B\|, c_{2}=$ $\hat{D} K\|A+B K\| \mathrm{e}^{\|A+B K\| \hat{D}}\|B\|, c_{3}=c_{2} /(\hat{D}\|B\|), c_{4}=$ $2 \hat{D} K \mathrm{e}^{\|A\| \hat{D}}, c_{5}=c_{4} / 2, c_{5}=2 \hat{D}^{2} K\|A B\| \mathrm{e}^{2\|A\| \hat{D}}$, and choose $b_{2} \geq 8|P B| / \lambda_{\min }(Q)$. Using the Cauchy-Schwartz and Young inequalities, we have:

$$
\begin{aligned}
& \dot{V}(t) \leq-\frac{\lambda_{\min }(Q)}{2}|X(t)|^{2}+\frac{b_{2}}{2}\left(\hat{w}(0, t)^{2}+\tilde{u}(0, t)^{2}\right) \\
& -2 b_{1}\left(\frac{\tilde{u}(0, t)^{2}}{2}+\frac{\|\tilde{u}(t)\|^{2}}{2}-\frac{|\tilde{D}|}{\hat{D}}\|\tilde{u}(t)\|^{2}\right. \\
& -\frac{|\tilde{D}|}{\hat{D}}\left\|\hat{w}_{x}(t)\right\|^{2}-|\tilde{D}| c_{1}^{2}\|\tilde{u}(t)\|^{2}-|\tilde{D}|\|\hat{w}(t)\|^{2} \\
& \left.-|\tilde{D}|\left(c_{2}^{2}\|\tilde{u}(t)\|^{2}+\|\hat{w}(t)\|\right)^{2}-|\tilde{D}|\left(|X(t)|^{2}+c_{3}^{2}\|\tilde{u}(t)\|^{2}\right)\right) \\
& -2 b_{2}\left(\frac{\hat{w}(0, t)^{2}}{2}+\frac{\|\hat{w}(t)\|^{2}}{2}-c_{4}^{2} \tilde{u}(0, t)^{2}-\frac{\|\hat{w}(t)\|^{2}}{4}\right) \\
& -2 b_{2}\left(\frac{\hat{w}_{x}(0, t)^{2}}{2}+\frac{\left\|\hat{w}_{x}(t)\right\|^{2}}{2}-c_{6}^{2} \tilde{u}(0, t)^{2}\right. \\
& \left.-\frac{\left\|\hat{w}_{x}(t)\right\|^{2}}{4}-c_{5}^{2} \tilde{u}(0, t)^{2}\right) .
\end{aligned}
$$


Grouping the like terms, we obtain

$$
\begin{aligned}
& \dot{V}(t) \leq-\left(\frac{\lambda_{\min }(Q)}{2}-2|\tilde{D}| b_{1}\right)|X(t)|^{2} \\
& -b_{1}\left(1-2|\tilde{D}|\left(\frac{1}{\hat{D}}+c_{1}^{2}+c_{2}^{2}+c_{3}^{2}\right)\right)\|\tilde{u}(t)\|^{2} \\
& -\left(b_{1}-2 b_{2}\left(\frac{1}{4}+c_{4}^{2}+c_{5}^{2}+c_{6}^{2}\right)\right) \tilde{u}(0, t)^{2} \\
& -\left(b_{2}-\frac{2|\tilde{D}|}{\hat{D}} b_{1}\right)\left\|\hat{w}_{x}(t)\right\|^{2}-\left(\frac{b_{2}}{2}-2 b_{1}|\tilde{D}|\right)\|\hat{w}(t)\|^{2} \\
& -\frac{b_{2}}{2} \hat{w}(0)^{2}-b_{2} \hat{w}_{x}(0, t)^{2}
\end{aligned}
$$

and, with some further majorizations (for $|\tilde{D}|<D$ ), we get

$$
\begin{aligned}
& \dot{V}(t) \leq-\left(\frac{\lambda_{\min }(Q)}{2}-2|\tilde{D}| b_{1}\right)|X(t)|^{2} \\
& -b_{1}\left(1-2 \frac{|\tilde{D}|\left(1+D\left(c_{1}^{2}+c_{2}^{2}+c_{3}^{2}\right)\right)}{D-|\tilde{D}|}\right)\|\tilde{u}(t)\|^{2} \\
& -\left(b_{1}-2 b_{2}\left(\frac{1}{4}+c_{4}^{2}+c_{5}^{2}+c_{6}^{2}\right)\right) \tilde{u}(0, t)^{2} \\
& -\left(b_{2}-\frac{2|\tilde{D}|}{D-|\tilde{D}|} b_{1}\right)\left\|\hat{w}_{x}(t)\right\|^{2}-\left(\frac{b_{2}}{2}-2 b_{1}|\tilde{D}|\right)\|\hat{w}(t)\|^{2} \\
& -\frac{b_{2}}{2} \hat{w}(0, t)^{2}-b_{2} \hat{w}_{x}(0)^{2}
\end{aligned}
$$

Assuming that $|\tilde{D}|<\delta^{*}$, where

$$
\delta^{*}=\min \left\{\frac{D}{3+2\left(c_{1}^{2}+c_{2}^{2}+c_{3}^{2}\right)}, \frac{D b_{2}}{4 b_{1}+b_{2}}, \frac{b_{2}}{4 b_{1}}, \frac{\lambda_{\min }(Q) D}{4 b_{1}}\right\}
$$

and $b_{1}>\frac{2}{1 / 4+c_{4}^{2}+c_{5}^{2}+c_{6}^{2}} b_{2}$, from (42) we obtain

$$
\begin{aligned}
\dot{V} \leq & -\eta\left(|X(t)|^{2}+\|\tilde{u}(t)\|^{2}+\tilde{u}(0, t)^{2}+\left\|\hat{w}_{x}(t)\right\|^{2}\right. \\
& \left.+\|\hat{w}(t)\|^{2}+\hat{w}(0, t)^{2}+\hat{w}_{x}(0, t)^{2}\right) \\
\leq & -\eta \Gamma_{0}
\end{aligned}
$$

where $\Gamma_{0}(t)=|X(t)|^{2}+\|\tilde{u}(t)\|^{2}+\|\hat{w}(t)\|^{2}+\left\|\hat{w}_{x}(t)\right\|^{2}$ and $\eta=\min \left\{\frac{\lambda_{\min }(Q)}{2}-2|\tilde{D}| b_{1}, b_{1}\left(1-2 \tilde{D}\left(\frac{1}{\hat{D}}+c_{1}^{2}+c_{2}^{2}+c_{3}^{2}\right)\right), b_{1}-\right.$ $\left.2 b_{2}\left(\frac{1}{4}+c_{4}^{2}+c_{5}^{2}+c_{6}^{2}\right), b_{2}-\frac{2|\tilde{D}|}{\hat{D}} b_{1}, \frac{b_{2}}{2}-2 b_{1}|\tilde{D}|, \frac{b_{2}}{2}\right\}$ is positive. Having obtained (46), to complete the proof of (39), we first obtain the following inequalities from (38):

$$
\begin{aligned}
V(t) \geq & \lambda_{\min }(P)|X(t)|^{2}+b_{1} D\|\tilde{u}(t)\|^{2} \\
& +b_{2} \hat{D}\left(\|\hat{w}(t)\|^{2}+\left\|\hat{w}_{x}(t)\right\|^{2}\right) \\
\geq & \min \left\{\lambda_{\min }(P), b_{1} D, b_{2} \hat{D}\right\} \Gamma_{0}(t) .
\end{aligned}
$$

From (46) and (48), we complete the proof of (39) with $\rho=$ $\frac{\eta}{\min \left\{\lambda_{\min }(P), b_{1} D, b_{2} \hat{D}\right\}}$.

Lemma 7: $\exists d_{1}, d_{2}>0$ such that $d_{1} \Gamma(t) \leq V(t) \leq d_{2} \Gamma(t)$.

Proof: From (26)-(29) we get

$$
\begin{aligned}
\Gamma(t) \leq & |X(t)|^{2}+2\left(\|\tilde{u}(t)\|^{2}+\left\|\tilde{u}_{x}(t)\right\|^{2}\right)+\|\hat{u}(t)\|^{2}+\left\|\hat{u}_{x}(t)\right\|^{2} \\
\leq & |X(t)|^{2}+2\|\tilde{u}(t)\|^{2}+3\left(p_{1}\|\hat{w}(t)\|^{2}+p_{2}|X(t)|^{2}\right) \\
& +4\left\|\hat{w}_{x}(t)\right\|^{2}+p_{3}\|\hat{w}(t)\|^{2}+p_{4}|X(t)|^{2} \\
\leq & \max \left\{1+3 p_{2}+p_{4}, 2,3 p_{1}+p_{3}, 4\right\} \Gamma_{0}(t)
\end{aligned}
$$

and

$$
\begin{aligned}
V(t) \leq & \lambda_{\max }(P)|X(t)|^{2}+2\left(\|\tilde{u}(t)\|^{2}+\|\hat{w}(t)\|^{2}+\left\|\hat{w}_{x}(t)\right\|^{2}\right) \\
\leq & \lambda_{\max }(P)|X(t)|^{2}+4\left(\|u(t)\|^{2}+\|\hat{u}\|^{2}\right) \\
& +2\left(q_{1}\|\hat{u}(t)\|^{2}+q_{2}|X(t)|^{2}\right) \\
& +2\left(4\left\|\hat{u}_{x}(t)\right\|^{2}+q_{3}\|\hat{u}(t)\|^{2}+q_{4}|X(t)|^{2}\right)
\end{aligned}
$$

so $\quad d_{1}=\frac{\max \left\{1+3 p_{2}+p_{4}, 3 p_{1}+p_{3}\right\}}{\min \left\{\lambda_{\min }(P), b_{1} D, b_{2} \hat{D}\right\}} \quad$ and $\quad d_{2}=$ $\max \left\{\lambda_{\max }(P)+2 q_{2}+2 q_{4}, 4+2 q_{1}+2 q_{3}\right\}$.

We now complete the proof of Theorem 1. From Lemma 6 it follows that $V(t) \leq V(0) \mathrm{e}^{-\rho t}$. From Lemma 7 we get $\Gamma(t) \leq \frac{d_{2}}{d_{1}} \Gamma(0) \mathrm{e}^{-\rho t}$. So $R=d_{2} / d_{1}$, which completes the proof of Theorem 1. Next we prove Corollary 2 .

Lemma 8: $\exists d_{3}, d_{4}>0$ such that $d_{3} \Gamma(t) \leq \Pi(t) \leq d_{4} \Gamma(t)$.

Proof: By substituting $u(x, t)=U(t+D(x-1))$ into (12), we get

$$
\begin{aligned}
\Gamma(t)= & |X(t)|^{2}+\frac{1}{D} \int_{t-D}^{t} U(\theta)^{2} d \theta+\frac{1}{\hat{D}} \int_{t-\hat{D}}^{t} U(\theta)^{2} d \theta \\
& +\hat{D} \int_{t-\hat{D}}^{t} \dot{U}(\theta)^{2} d \theta
\end{aligned}
$$

Thus, $d_{3}=\frac{1}{2 \max \left\{1, \frac{1}{D}, \frac{1}{\hat{D}}, \hat{D}\right\}}, d_{4}=\max \left\{1, D, \hat{D}, \frac{1}{\hat{D}}\right\}$.

By combining Theorem 1 with Lemma 8, we complete the proof of Corollary 2 with $R^{\prime}=\frac{d_{4}}{d_{3}} R$.

\section{Adaptive Control With Estimation of the TRANSPORT PDE STATE}

Now we establish local stability for an adaptive controller. A global result is not obtainable because the solution $u(x, t)=$ $U(t+D(x-1))$ is not linearly parametrizable in $D$.

Assumption 1: A lower bound $\underline{D}>0$ and an upper bound $\bar{D} \geq \underline{D}$ on the unknown delay $D$ are known.

The update law for $\hat{D}$ is chosen as

$$
\begin{aligned}
\dot{\hat{D}}(t) & =\gamma \operatorname{Proj}_{[\underline{D} ; \bar{D}]}\{\tau(t)\}, \quad \gamma>0 \\
\tau(t) & =-\int_{0}^{1}(1+x) \hat{w}(x, t) K \mathrm{e}^{A \hat{D}(t) x} d x(A X(t)+B \hat{u}(0, t)
\end{aligned}
$$

where the standard projection operator is given by

$$
\operatorname{Proj}_{[\underline{D}, \bar{D}]}\{\tau\}= \begin{cases}0, & \hat{D}=\underline{D} \text { and } \tau<0 \\ 0, & \hat{D}=\bar{D} \text { and } \tau>0 \\ \tau, & \text { else. }\end{cases}
$$

Theorem 9: Consider the closed loop consisting of the plant (2)-(4), (6), (7), the control law (8), and the update law defined by (54)-(9). Let Assumption 1 hold and let

$$
\begin{aligned}
\Upsilon(t)= & |X(t)|^{2}+\int_{0}^{1} u(x, t)^{2} d x+\int_{0}^{1} \hat{u}(x, t)^{2} d x \\
& +\int_{0}^{1} \hat{u}_{x}(x, t)^{2} d x+\tilde{D}(t)^{2}
\end{aligned}
$$

denote the norm of the overall state of the closed-loop system. There exist positive constants $\rho$ and $R$ such that if the initial state $\left(X_{0}, u_{0}, \hat{u}_{0}, \hat{D}_{0}\right)$ is such that $\Upsilon(0)<\rho$, then

$$
\begin{gathered}
\Upsilon(t) \leq R \Upsilon(0), \\
\lim _{t \rightarrow \infty} X(t)=0, \quad \lim _{t \rightarrow \infty} U(t)=0 .
\end{gathered}
$$


Proof: Mimicking the (omitted) proof of Lemma 3, we obtain the $(X, \tilde{u}, \hat{w})$-system as

$$
\begin{aligned}
\dot{X}(t)= & (A+B K) X(t)+B \hat{w}(0, t)+B \tilde{u}(0, t) \\
D \tilde{u}_{t}(x, t)= & \tilde{u}_{x}(x, t)-\tilde{D}(t) r(x, t) \\
& -D \dot{\hat{D}}(t)(x-1) r(x, t) \\
\tilde{u}(1, t)= & 0 \\
\hat{D}(t) \hat{w}_{t}(x, t)= & \hat{w}_{x}(x, t)-\hat{D}(t) \dot{\hat{D}}(t) s(x, t) \\
& -\hat{D}(t) K \mathrm{e}^{A \hat{D}(t) x} B \tilde{u}(0, t) \\
\hat{w}(1, t)= & 0
\end{aligned}
$$

where

$$
\begin{aligned}
r(x, t)= & \frac{\hat{w}_{x}(x, t)}{\hat{D}(t)}+K B \hat{w}(x, t) \\
& +\int_{0}^{x} K(A+B K) \hat{D}(t) \mathrm{e}^{(A+B K) \hat{D}(t)(x-y)} B \hat{w}(y, t) d y \\
& +K(A+B K) \mathrm{e}^{(A+B K) \hat{D}(t) x} X(t) \\
s(x, t)= & (1-x)\left(\frac{\hat{w}_{x}(x, t)}{\hat{D}(t)}+K \mathrm{e}^{A \hat{D}(t) x} B(K X(t)+\hat{w}(0, t))\right) \\
& +\int_{0}^{x} \hat{w}(y, t)\left[K(I+A \hat{D}(t)(x-y)) \mathrm{e}^{A \hat{D}(t)(x-y)} B\right. \\
& +\hat{D}(t) \int_{y}^{x} K(I+A \hat{D}(t)(x-\xi)) \mathrm{e}^{A \hat{D}(t)(x-\xi)} \\
& \left.\times B K \mathrm{e}^{(A+B K) \hat{D}(t)(\xi-y)} B d \xi\right] d y \\
& +\left(K A \mathrm{e}^{A \hat{D}(t) x}+\int_{0}^{x} K(I+A \hat{D}(t)(x-y))\right. \\
& \left.\times \mathrm{e}^{A \hat{D}(t)(x-y)} B K \mathrm{e}^{(A+B K) \hat{D}(t) y} d y\right) X(t) .
\end{aligned}
$$

Since our Lyapunov analysis will involve an $H_{1}$ norm of $\hat{w}$, we also need the governing equations of the $\hat{w}_{x}$-system:

$$
\begin{aligned}
\hat{D}(t) \hat{w}_{x t}(x, t)= & \hat{w}_{x x}(x, t)-\hat{D} \dot{\hat{D}}(t) s_{x}(x, t) \\
& -K A \hat{D}^{2} \mathrm{e}^{A \hat{D}(t) x} B \tilde{u}(0, t) \\
\hat{w}_{x}(1, t)= & \hat{D}(t) \dot{\hat{D}}(t)\left(\left[K A \mathrm{e}^{A \hat{D}(t)}+\int_{0}^{1} K(I+A \hat{D}(t)(1-y))\right.\right. \\
& \left.\times \mathrm{e}^{A \hat{D}(t)(1-y)} B K \mathrm{e}^{(A+B K) \hat{D}(t) y} d y\right] X(t) \\
& +\int_{0}^{1} \hat{w}(y, t)\left[K(I+A \hat{D}(t)(1-y)) \mathrm{e}^{A \hat{D}(t)(1-y)}\right. \\
& +\int_{y}^{1} K(I+A \hat{D}(t)(1-\xi)) \mathrm{e}^{A \hat{D}(t)(1-\xi)} \\
& \left.\left.\times B K \mathrm{e}^{(A+B K) \hat{D}(t)(\xi-y)} d \xi\right] B d y\right) \\
+ & \hat{D}(t) K \mathrm{e}^{A \hat{D}(t)} B \tilde{u}(0, t)
\end{aligned}
$$

where

$$
\begin{aligned}
& s_{x}(x, t)=\frac{1}{\hat{D}(t)}\left[(1-x) \hat{w}_{x x}(x, t)-\hat{w}_{x}(x, t)\right] \\
& +K \mathrm{e}^{A \hat{D}(t) x}(A \hat{D}(t)(1-x)-I) B(K X(t)+\hat{w}(0, t)) \\
& +\hat{D}(t) \int_{0}^{x} \hat{w}(y, t)[K(B K+A(2 I \\
& +A \hat{D}(t)(x-y))) \mathrm{e}^{A \hat{D}(t)(x-y)} B \\
& +\hat{D}(t) \int_{y}^{x} K(B K+A(2 I+A \hat{D}(t)(x-\xi)))
\end{aligned}
$$

$$
\begin{aligned}
& \left.\times \mathrm{e}^{A \hat{D}(t)(x-\xi)} B K \mathrm{e}^{(A+B K) \hat{D}(t)(\xi-y)} B d \xi\right] d y \\
& +K B \hat{w}(x, t)+\left[K\left(A^{2} \hat{D}(t)+B K\right) \mathrm{e}^{A \hat{D}(t) x}\right. \\
& +\hat{D}(t) \int_{0}^{x} K(B K+A(2 I+A \hat{D}(t)(x-y))) \\
& \left.\times \mathrm{e}^{A \hat{D}(t)(x-y)} B K \mathrm{e}^{(A+B K) \hat{D}(t) y} d y\right] X(t) .
\end{aligned}
$$

We now start our Lyapunov analysis by introducing

$$
\begin{aligned}
V(t)= & X^{T}(t) P X(t)+b_{1} D \int_{0}^{1}(1+x) \tilde{u}(x, t)^{2} d x \\
& +b_{2} \hat{D}(t) \int_{0}^{1}(1+x)\left(\hat{w}(x, t)^{2}+\hat{w}_{x}(x, t)^{2}\right) d x+b_{3} \tilde{D}(t)^{2} .
\end{aligned}
$$

With (60)-(69), we get

$$
\begin{aligned}
& \dot{V}(t)=-X^{T}(t) Q X(t)+2 X^{T}(t) P B(\hat{w}(0, t)+\tilde{u}(0, t)) \\
& +b_{1}\left(-\tilde{u}(0, t)^{2}-\|\tilde{u}(t)\|^{2}-2 \tilde{D}(t) \int_{0}^{1}(1+x) \tilde{u}(x, t) r(x, t) d x\right. \\
& \left.-2 D \dot{\hat{D}}(t) \int_{0}^{1}\left(x^{2}-1\right) \tilde{u}(x, t) r(x, t) d x\right) \\
& +b_{2}\left(-\hat{w}(0, t)^{2}-\|\hat{w}(t)\|^{2}\right. \\
& -2 \hat{D}(t) \dot{\hat{D}}(t) \int_{0}^{1}(1+x) \hat{w}(x, t) s(x, t) d x \\
& \left.-2 \hat{D}(t) \int_{0}^{1}(1+x) K \mathrm{e}^{A \hat{D}(t) x} B \tilde{u}(0, t) \hat{w}(x, t) d x\right) \\
& +b_{2}\left(2 \hat{w}_{x}(1, t)^{2}-\hat{w}_{x}(0, t)^{2}-\left\|\hat{w}_{x}(t)\right\|^{2}\right. \\
& -2 \hat{D}(t) \dot{\hat{D}}(t) \int_{0}^{1}(1+x) \hat{w}_{x}(x, t) s_{x}(x, t) d x \\
& \left.-2 \hat{D}(t)^{2} \int_{0}^{1}(1+x) A K \mathrm{e}^{A \hat{D}(t) x} B \tilde{u}(0, t) \hat{w}_{x}(x, t) d x\right) \\
& +\dot{\hat{D}}(t) b_{2}\left(\int_{0}^{1}(1+x) \hat{w}(x, t)^{2} d x\right. \\
& \left.+\int_{0}^{1}(1+x) \hat{w}_{x}(x, t)^{2} d x\right)-2 b_{3} \tilde{D}(t) \dot{\hat{D}}(t) .
\end{aligned}
$$

Using (54), (65), (66), (68), (69), the properties of the projection operator, and Agmon's inequality $\hat{w}(0, t)^{2} \leq$ $4\left\|\hat{w}_{x}(t)\right\|^{2}$ (with the fact that $\hat{w}(1, t)=0$ ), we find constants $M_{1}, M_{2}, \ldots, M_{8}$ (independent of initial conditions) such that

$$
\begin{array}{r}
\left|\int_{0}^{1}(1+x) \tilde{u}(x, t) r(x, t) d x\right| \leq \\
M_{1}\left(\|\tilde{u}(t)\|^{2}+\left\|\hat{w}_{x}(t)\right\|^{2}+\|\hat{w}(t)\|^{2}+|X(t)|^{2}\right) \\
\left|\int_{0}^{1}\left(x^{2}-1\right) \tilde{u}(x, t) r(x, t) d x\right| \leq \\
M_{1}\left(\|\tilde{u}(t)\|^{2}+\left\|\hat{w}_{x}(t)\right\|^{2}+\|\hat{w}(t)\|^{2}+|X(t)|^{2}\right) \\
\left|2 \hat{D}(t) \int_{0}^{1}(1+x) \hat{w}(x, t) s(x, t) d x\right| \leq \\
4 M_{2}\left(\|\hat{w}(t)\|^{2}+\left\|\hat{w}_{x}(t)\right\|^{2}+|X(t)|^{2}\right) \\
\int_{0}^{1}(1+x) K \mathrm{e}^{A \hat{D}(t) x} B \tilde{u}(0, t) \hat{w}(x, t) d x \mid \leq \\
M_{3} \tilde{u}(0, t)^{2}+\frac{\| w \hat{w} t) \|^{2}}{4} \\
\left|2 \hat{D}(t) \int_{0}^{1}(1+x) \hat{w}_{x}(x, t) s_{x}(x, t) d x\right| \leq
\end{array}
$$




$$
\begin{array}{r}
4 M_{4}\left(\|\hat{w}(t)\|^{2}+\left\|\hat{w}_{x}(t)\right\|^{2}+|X(t)|^{2}\right) \\
\left|2 \hat{D}(t)^{2} \int_{0}^{1}(1+x) A K \mathrm{e}^{A \hat{D}(t) x} B \tilde{u}(0, t) \hat{w}_{x}(x, t) d x\right| \leq \\
M_{5} \tilde{u}(0, t)^{2}+\frac{\left\|\hat{w}_{x}(t)\right\|^{2}}{4} \\
|\dot{\hat{D}}(t)| \leq 4 M_{6}\left(|X(t)|^{2}+\|\hat{w}(t)\|^{2}+\left\|\hat{w}_{x}(t)\right\|^{2}\right) \\
2 \hat{w}_{x}(1, t)^{2} \leq \dot{\hat{D}}(t)^{2} M_{7}\left(|X(t)|^{2}+\|\hat{w}(t)\|^{2}\right)+M_{8} \tilde{u}(0, t)^{2} .
\end{array}
$$

Then,

$$
\begin{aligned}
& \dot{V}(t) \leq-\frac{\lambda_{\min }(Q)}{2}|X(t)|^{2}-\frac{b_{2}}{2} \hat{w}(0, t)^{2}-b_{2} \hat{w}_{x}(0, t)^{2} \\
& -\left(b_{2}-\frac{b_{2}}{4}\right)\|\hat{w}(t)\|^{2}-\left(b_{2}-\frac{b_{2}}{4}\right)\left\|\hat{w}_{x}(t)\right\|^{2} \\
& -b_{1}\|\tilde{u}(t)\|^{2}-\left(b_{1}-b_{2}\left(\frac{1}{2}+M_{3}+M_{5}+M_{8}\right)\right) \tilde{u}(0, t)^{2} \\
& +b_{2} M_{7} \dot{\hat{D}}(t)^{2}\left(|X(t)|^{2}+\|\hat{w}(t)\|^{2}\right)+2 b_{3}|\tilde{D}(t)||\hat{\hat{D}}(t)| \\
& +b_{1} M_{1} \bar{D}|\dot{\hat{D}}(t)|\left(\|\tilde{u}(t)\|^{2}+\left\|\hat{w}_{x}(t)\right\|^{2}+\|\hat{w}(t)\|^{2}+|X(t)|^{2}\right) \\
& +b_{1} M_{1}|\tilde{D}(t)|\left(\|\tilde{u}(t)\|^{2}+\left\|\hat{w}_{x}(t)\right\|^{2}+\|\hat{w}(t)\|^{2}+|X(t)|^{2}\right) \\
& +|\dot{\hat{D}}(t)|\left(4 b_{2} M_{2}\left(\|\hat{w}(t)\|^{2}+\left\|\hat{w}_{x}(t)\right\|^{2}+|X(t)|^{2}\right)\right. \\
& +4 b_{2} M_{4}\left(\|\hat{w}(t)\|^{2}+\left\|\hat{w}_{x}(t)\right\|^{2}+|X(t)|^{2}\right) \\
& \left.+2 b_{2}\left(\|\hat{w}(t)\|^{2}+\left\|\hat{w}_{x}(t)\right\|^{2}\right)\right),
\end{aligned}
$$

where we have chosen $b_{2} \geq 8|P B| / \lambda_{\min }(Q)$. By choosing $b_{1}>b_{2}\left(1 / 2+M_{3}+M_{5}+M_{8}\right)$, and defining $\eta=\min \left\{\frac{\lambda_{\min }(Q)}{2}, b_{2} / 2, b_{1}-b_{2}\left(1 / 2+M_{3}+M_{5}+M_{8}\right)\right\}$ and $V_{0}(t)=|X(t)|^{2}+\|\tilde{u}(t)\|^{2}+\|\hat{w}(t)\|^{2}+\left\|\hat{w}_{x}(t)\right\|^{2}$, where $\eta>0$,

$$
\begin{aligned}
\dot{V}(t) \leq & -\eta\left(|X(t)|^{2}+\|\tilde{u}(t)\|^{2}+\|\hat{w}(t)\|^{2}+\left\|\hat{w}_{x}(t)\right\|^{2}\right. \\
& \left.+\tilde{u}(0, t)^{2}+\hat{w}(0, t)^{2}+\hat{w}_{x}(0, t)\right) \\
& +16 b_{2} M_{7} M_{6}^{2} V_{0}(t)^{3}+4 b_{1} M_{1} M_{6} \bar{D} V_{0}(t)^{2} \\
& +\left(8 b_{3} M_{6}+b_{1} M_{1}\right)|\tilde{D}(t)| V_{0}(t) \\
& +4 M_{6}\left(4 b_{2} M_{2}+4 b_{2} M_{4}+2 b_{2}\right) V_{0}(t)^{2} \\
\leq \quad & -\eta V_{0}(t)+\left(8 b_{3} M_{6}+b_{1} M_{1}\right)|\tilde{D}(t)| V_{0}(t) \\
& +4 M_{6}\left(b_{1} M_{1} \bar{D}+2 b_{2}\left(2 M_{2}+2 M_{4}+1\right) V_{0}(t)^{2}\right. \\
& +16 b_{2} M_{7} M_{6}^{2} V_{0}(t)^{3}
\end{aligned}
$$

To eliminate the parameter error term we employ the bound

$$
\begin{aligned}
|\tilde{D}(t)| \leq & \frac{\varepsilon}{2}+\frac{\tilde{D}(t)^{2}}{2 \varepsilon} \\
\leq & \frac{\varepsilon}{2}+\frac{1}{2 b_{3} \varepsilon}\left(V(t)-\lambda_{\min }(P)|X(t)|^{2}-b_{1} \underline{D}\|\tilde{u}(t)\|^{2}\right. \\
& \left.-b_{2} \underline{D}\|\hat{w}(t)\|^{2}-b_{2} \underline{D}\left\|\hat{w}_{x}(t)\right\|^{2}\right) \\
\leq & \frac{\varepsilon}{2}+\frac{1}{2 b_{3} \varepsilon}\left(V(t)-\min \left\{\lambda_{\min }(P), b_{1} \underline{D}, b_{2} \underline{D},\right\} V_{0}(t)\right),
\end{aligned}
$$

which yields

$$
\begin{aligned}
\dot{V}(t) \leq & -\left(\eta-\left(8 b_{3} M_{6}+b_{1} M_{1}\right)\left(\frac{\varepsilon}{2}+\frac{1}{2 b_{3} \varepsilon} V(t)\right)\right) V_{0}(t) \\
& -\left(\frac{\left(8 b_{3} M_{6}+b_{1} M_{1}\right) \min \left\{\lambda_{\min }(P), b_{1} \underline{D}, b_{2} \underline{D}\right\}}{2 b_{3} \varepsilon}\right. \\
& -4 M_{6}\left(b_{1} M_{1} \bar{D}+2 b_{2}\left(2 M_{2}+2 M_{4}+1\right)\right)
\end{aligned}
$$

$$
\left.-16 b_{2} M_{7} M_{6}^{2} V_{0}(t)\right) V_{0}(t)^{2} .
$$

If we choose the analysis parameter $\varepsilon$ as

$$
\begin{aligned}
& \varepsilon \leq \min \left\{\frac{2 \eta}{8 b_{3} M_{6}+b_{1} M_{1}},\right. \\
& \left.\frac{\left(8 b_{3} M_{6}+b_{1} M_{1}\right) \min \left\{\lambda_{\min }(P), b_{1} \underline{D}, b_{2} \underline{D}\right\}}{8 b_{3} M_{6}\left(b_{1} M_{1} \bar{D}+2 b_{2}\left(2 M_{2}+2 M_{4}+1\right)\right)}\right\}
\end{aligned}
$$

and restrict the initial conditions so that $V(0) \leq \rho_{1}$,

$$
\begin{aligned}
\rho_{1} \triangleq & \min \left\{2 b_{3} \varepsilon\left(\frac{\eta}{8 b_{3} M_{6}+b_{1} M_{1}}-\frac{\varepsilon}{2}\right),\right. \\
& \frac{\min \left\{\lambda_{\min }(P), b_{1} \underline{D}, b_{2} \underline{D}\right\}}{16 b_{2} M_{7} M_{6}^{2}} \\
& \times\left(\frac{\left(8 b_{3} M_{6}+b_{1} M_{1}\right) \min \left\{\lambda_{\min }(P), b_{1} \underline{D}, b_{2} \underline{D}\right\}}{2 b_{3} \varepsilon}\right. \\
& \left.\left.-4 M_{6}\left(b_{1} M_{1} \bar{D}+2 b_{2}\left(2 M_{2}+2 M_{4}+1\right)\right)\right)\right\},
\end{aligned}
$$

we obtain $\dot{V}(t) \leq-\mu_{1}(t) V_{0}(t)-\mu_{2}(t) V_{0}(t)^{2}$, where

$$
\begin{aligned}
\mu_{1}(t)= & \eta-\left(8 b_{3} M_{6}+b_{1} M_{1}\right)\left(\frac{\varepsilon}{2}+\frac{1}{2 b_{3} \varepsilon} V(t)\right) \\
\mu_{2}(t)= & \frac{\left(8 b_{3} M_{6}+b_{1} M_{1}\right) \min \left\{\lambda_{\min }(P), b_{1} \underline{D}, b_{2} \underline{D}\right\}}{2 b_{3} \varepsilon} \\
& -4 M_{6}\left(b_{1} M_{1} \bar{D}+2 b_{2}\left(2 M_{2}+2 M_{4}+1\right)\right) \\
& -\frac{16 b_{2} M_{7} M_{6}^{2}}{\min \left\{\lambda_{\min }(P), b_{1} \underline{D}, b_{2} \underline{D}\right\}} V(t)
\end{aligned}
$$

are nonnegative functions if the initial conditions are as in (84). Hence, $V(t) \leq V(0), \forall t \geq 0$. From this result for $V(t)$, we the result for $\Upsilon(t)$. Using Lemma 5 (which holds both when $\hat{D}$ is constant and with a time-varying $\hat{D}(t)$ ), we obtain

$$
\begin{aligned}
\Upsilon(t)= & |X(t)|^{2}+\|u(t)\|^{2}+\|\hat{u}(t)\|^{2}+\left\|\hat{u}_{x}(t)\right\|^{2}+\tilde{D}(t)^{2} \\
\leq & |X(t)|^{2}+2\left(\|\tilde{u}(t)\|^{2}+\|\hat{u}(t)\|^{2}\right) \\
& +\|\hat{u}(t)\|^{2}+\left\|\hat{u}_{x}(t)\right\|^{2}+\tilde{D}(t)^{2} \\
\leq & \left(1+3 p_{2}+p_{4}\right)|X(t)|^{2}+2\|\tilde{u}(t)\|^{2} \\
& +\left(3 p_{1}+p_{3}\right)\|\hat{w}(t)\|^{2}+4\left\|\hat{w}_{x}(t)\right\|^{2}+\tilde{D}(t)^{2} \\
\leq & \max \left\{1+3 p_{2}+p_{4}, 3 p_{1}+p_{3}, 4\right\} \\
& \times\left(|X(t)|^{2}+\|\tilde{u}(t)\|^{2}+\|\hat{w}(t)\|^{2}\right. \\
& \left.+\left\|\hat{w}_{x}(t)\right\|^{2}+\tilde{D}(t)^{2}\right) \\
\leq & \left.\frac{\max \left\{1+3 p_{2}+p_{4}, 3 p_{1}+p_{3}\right\}}{\min \left\{\lambda_{\min }(P), b_{1} D, b_{2} D\right.}, b_{3}\right\} \\
\triangleq & \rho_{2} V(t) \leq \rho_{2} V(0) .
\end{aligned}
$$

Hence, from (84) and (87) we obtain $\rho=\rho_{1} \rho_{2}$. Similarly, using Lemma 5, we show that

$$
\begin{aligned}
V(0) \leq & \max \left\{\lambda_{\max }(P), 2 b_{1} \bar{D}, 2 b_{2} \bar{D}, b_{3}\right\} \\
& \times\left(|X(t)|^{2}+\|\tilde{u}(t)\|^{2}+\|\hat{w}(t)\|^{2}+\left\|\hat{w}_{x}(t)\right\|^{2}\right) \\
\leq & \max \left\{\lambda_{\max }(P), 2 b_{1} \bar{D}, 2 b_{2} \bar{D}, b_{3}\right\} \\
& \times \max \left\{1+q_{2}+q_{4}, 2,2+q_{1}+q_{3}, 4\right\} \Upsilon(0) .
\end{aligned}
$$

Then, using (87), (88), we complete the local stability proof with $R=\frac{\max \left\{1+3 p_{2}+p_{4}, 3 p_{1}+p_{3}\right\}}{\min \left\{\lambda_{\min }(P), b_{1} \underline{D}, b_{2} \underline{D}, b_{3}\right\}} \max \left\{\lambda_{\max }(P), 2 b_{1} \bar{D}, 2 b_{2} \bar{D}, b_{3}\right\}$ $\max \left\{1+q_{2}+q_{4}, 2+q_{1}+q_{3}\right\}$. To prove regulation, from 
$V(t) \leq V(0)$ it follows that $X(t),\|\tilde{u}\|,\|\hat{w}\|,\left\|\hat{w}_{x}\right\|, \hat{D}(t)$ are uniformly bounded. Then, from (10), using Cauchy-Schwartz inequality, we obtain the uniform boundedness of $\|\hat{u}(t)\|$ and consequently also of $U(t)$ for $t \geq 0$ from (8). Thus $u(0, t)=$ $U(t-D)$ is uniformly bounded for $t \geq D$. Using (2), we get that $d|X(t)|^{2} / d t$ is uniformly bounded for $t \geq D$. From (82), it follows that $|X(t)|$ is square integrable. Finally, by Barbalat's lemma, we get that $X(t) \rightarrow 0$. To also prove the regulation of $U(t)$, we start by deducing from (82) the square integrability of $\|\hat{w}(t)\|$. Then, from Lemma 5, we have the square integrability of $\|\hat{u}\|$ and, from (8), using Cauchy-Schwartz inequality, the square integrability of $U(t)$. To establish the boundedness of $d U(t)^{2} / d t$, we compute it as

$$
\begin{aligned}
\frac{d}{d t} U(t)^{2}= & 2 U(t) K\left(\mathrm{e}^{A \hat{D}(t)} \dot{X}(t)+\dot{\hat{D}}(t) G_{1}(t)+\hat{D}(t) G_{2}(t)\right) \\
G_{1}(t)= & A \mathrm{e}^{A \hat{D}(t)} X(t)+(x-1) \int_{0}^{1} \mathrm{e}^{A \hat{D}(t)(1-y)} B \hat{u}_{x}(y, t) d y \\
& +\int_{0}^{1}(I+A \hat{D}(t)(1-y)) \mathrm{e}^{A \hat{D}(t)(1-y)} B \hat{u}(y, t) d y \\
G_{2}(t)= & \int_{0}^{1} \mathrm{e}^{A \hat{D}(t)(1-y)} B \hat{u}_{x}(y, t) d y
\end{aligned}
$$

The signal $\dot{\hat{D}}(t)$ is uniformly bounded for $t \geq D$ according to (54). By using the boundedness of $\dot{X}(t), X(t),\|\hat{u}(t)\|$ and $\left\|\hat{u}_{x}(t)\right\|$ over $t \geq D$, we get boudedness of $d U(t)^{2} / d t$ for $t \geq D$. Then, by Barbalat's lemma, $U(t) \rightarrow 0$ as $t \rightarrow \infty$.

\section{Simulations}

Consider the system $X(s) / U(s)=\mathrm{e}^{-D s} B /(s-A)$, which is a model of the dynamics of an X-29 aircraft in an unstable regime [2], with the input being the control surface deflection, and with the output being a linear combination of the pitch rate (measured with a gyroscope) and the rate of change of the flight path (measured with a gyroscope). We take the plant parameters as $A=0.75, B=1$, and the nominal control gain as $K=-A-1=-1.75$ (which means that $P=1$ for $Q=2$ ). Figure 2 shows a simulation example with $D=1, A=0.5, B=$ $1, K=-1.5, P=1, Q=2$, and $\gamma=5$.

\section{RELATED Results}

In this paper the only parametric uncertainty considered is the unknown delay. In a companion paper [1] we present an extension with unknown plant parameters and where the control objective is not regulation to zero but trajectory tracking. The design technique in this paper is inspired by the techniques for parabolic PDEs in [7] and the non-adaptive techniques for hyperbolic PDEs [8]. Nonlinear extensions of predictor feedback are introduced in [5].

\section{REFERENCES}

[1] D. Bresch-Pietri and M. Krstic, "Adaptive trajectory tracking despite unknown actuator delay and plant parameters," Automatica, vol. 45, pp. 2075-2081, 2009.

[2] D. Ens, H. Ozbay, and A. Tannenbaum, "Abstract model and controller design for an unstable aircraft," Journal of Guidance, Control, and Dynamics, vol. 15, pp. 498-508, 1992.

[3] S. Evesque, A. M. Annaswamy, S. Niculescu, and A. P. Dowling, "Adaptive Control of A Class of Time-delay Systems," ASME Trans. Dynamics Syst., Meas. Control, vol. 125, pp. 186-193, 2003.

[4] M. Krstic, "Lyapunov tools for predictor feedbacks for delay systems: Inverse optimality and robustness to delay mismatch," Automatica, vol. 44, pp. 2930-2935, 2008
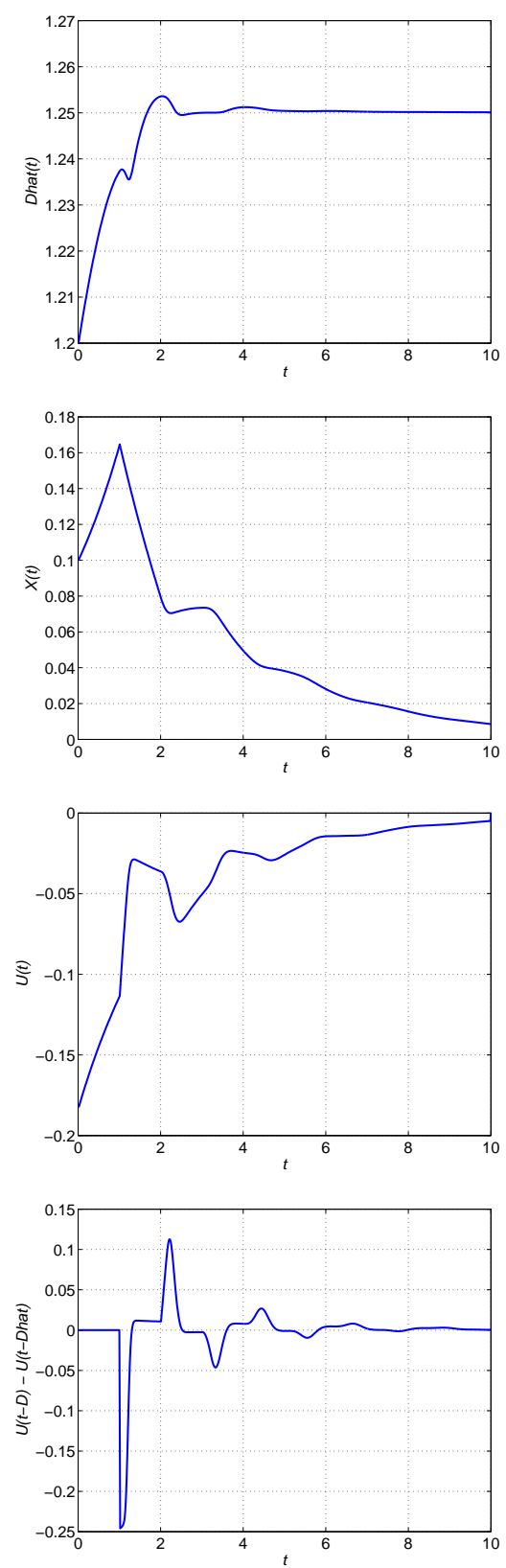

Fig. 2. The system response of the system (2)-(4), (6), (7), (8)-(9) for $D=1$. Bottom: the estimation error of the actuator state, $\tilde{u}(0, t)=u(0, t)-\hat{u}(0, t)=$ $U(t-D)-U(t-\hat{D}(t))$.

[5] M. Krstic, "Input delay compensation for forward complete and feedforward nonlinear systems, IEEE Transactions on Automatic Control, vol. 55, pp. 287-303, 2010.

[6] M. Krstic and D. Bresch-Pietri, "Delay-adaptive full-state predictor feedback for systems with unknown long actuator delay," 2009 American Control Conference.

[7] M. Krstic and A. Smyshlyaev, "Adaptive boundary control for unstable parabolic PDEs-Part I: Lyapunov design," IEEE Transactions on Automatic Control, vol. 53, pp. 1575-1591, 2008.

[8] M. Krstic and A. Smyshlyaev, "Backstepping boundary control for first order hyperbolic PDEs and application to systems with actuator and sensor delays," Syst. Contr. Let., vol. 57, pp. 750-758, 2008.

[9] S.-I. Niculescu and A. M. Annaswamy, "An Adaptive Smith-Controller for Time-delay Systems with Relative Degree $n^{*} \geq 2$," Systems \& Control Letters, vol. 49, pp. 347-358, 2003.

[10] R. Ortega and R. Lozano, "Globally stable adaptive controller for systems with delay," Internat. J. Control, vol. 47, pp. 17-23, 1988. 